\title{
Gastroenterology Consultations in Pregnancy
}

\author{
Sumona Saha, M.D., Joseph Manlolo, M.D., ${ }^{2}$ Christopher E. McGowan, M.D., ${ }^{3}$ \\ Steven Reinert, M.S., ${ }^{4}$ and Silvia Degli Esposti, M.D. ${ }^{5}$
}

\begin{abstract}
Background: Training in gastrointestinal (GI) disorders in pregnancy is required for all gastroenterology fellows. Nevertheless, the actual role of the gastroenterologist in the management of pregnant patients is unknown. Establishing the characteristics of GI consultations in pregnancy can help focus trainee education and prepare gastroenterologists for future practice. The purpose of this study was to determine the indications for consultations in pregnancy and the gastroenterologist's role in the evaluation and management of the pregnant patient. Methods: A chart review was performed of all consecutive outpatient GI consultations for pregnant women at a high-volume obstetrics hospital over a 3-year period. Referring source, patient characteristics, indication(s) for consultation, diagnosis(es), change in management after consultation, and need for follow-up were recorded.

Results: We reviewed 370 charts. The mean age ( \pm standard deviation [SD]) at referral was 28.7 years \pm 6.5 , and mean weeks of gestation $( \pm \mathrm{SD})$ was $21.3 \pm 8.8$. Obstetrician/gynecologists requested most consultations $(70.1 \%)$. New GI symptoms arising in pregnancy comprised $35.4 \%$ of consultations, and worsening of a preexisting GI disorder comprised $24.4 \%$. The most common indications for consultation were viral hepatitis $(20.2 \%)$, nausea and vomiting $(18.9 \%)$, and nonspecific abdominal pain $(13.5 \%)$. The most common diagnoses were acute or chronic viral hepatitis $(17.8 \%)$, hyperemesis gravidarum $(15.1 \%)$, gastroesophageal reflux disease $(14.3 \%)$, and constipation $(13.0 \%)$. Consultation changed the diagnosis in $25.1 \%$ of patients and changed management in $78.6 \%$. Follow-up was required in $77.3 \%$ of cases during pregnancy and $37.8 \%$ postpartum.

Conclusions: GI consultation in pregnancy is sought more frequently for the evaluation and management of GI disorders not unique to pregnancy than for pregnancy-unique disorders. Although GI consultation changed the diagnosis in a minority of cases, it changed management in the majority. Gastroenterologists should be familiar with the most common indications for consultation in pregnancy and be prepared to evaluate and manage pregnant women with GI disorders.
\end{abstract}

\section{Introduction}

O BSTETRICIANS ARE TRADITIONALLY VIEWED as the main providers of medical care to pregnant women. Although many medical conditions arising in pregnancy can be managed by obstetricians alone, some problems are beyond their scope of training and experience. Furthermore, with national statistics showing a trend toward delayed childbearing ${ }^{1}$ and improvements in medical and surgical management allowing women with chronic medical illness to conceive, the need for medical consultants in the care of pregnant women is increasing. ${ }^{2}$

Maternal/fetal medicine specialists and obstetric medicine internists, where available, often take on the responsibility of managing the medically complicated pregnancy. However, given the rapid advancements in diagnostic techniques and pharmacotherapeutics in each subfield of internal medicine, internal medicine subspecialists may be called upon to lend expertise in the care of the pregnant woman. To prepare for this role, they must know the indications for referral to their specialty during pregnancy and how to offer accurate and safe diagnostic and therapeutic recommendations.

In the gastrointestinal (GI) tract, normal physiological changes during pregnancy produce dramatic modifications. Visceral organs rearrange to accommodate uterine growth. Ovarian and placental hormone levels fluctuate, altering esophageal sphincter pressure, ${ }^{3,4}$ GI motility, ${ }^{5}$ gallbladder contractility, ${ }^{5}$ and intrahepatic bile salt transport. In addition,

\footnotetext{
${ }^{1}$ University of Wisconsin School of Medicine and Public Health, Madison, Wisconsin.

${ }^{2}$ Stony Brook University Medical Center, Stony Brook, New York.

${ }^{3}$ University of North Carolina, Chapel Hill, North Carolina.

${ }^{4}$ Lifespan Information Services, Providence, Rhode Island.

${ }^{5}$ The Warren Alpert Medical School of Brown University, Providence, Rhode Island.
} 
immunological changes, such as maternal/fetal cell trafficking $^{7}$ and maternal peripheral tolerance, ${ }^{8}$ occur. These may produce adverse, beneficial, or neutral effects in the pregnant mother with regard to autoimmune and infectious disease. Pregnancy, therefore, poses a unique medical stress to the GI tract, and for many women, is a period of vulnerability for the development of new or exacerbation of existing GI disorders.

The indications for GI consultation in pregnancy are currently unknown. Establishing these indications, however, is important for providing a focus for education for gastroenterologists and primary care providers who contribute to the care of the medically compromised pregnant woman. Focused education with enhanced understanding of these conditions will minimize the discomfort many nonobstetrician physicians experience when confronted with a pregnant patient with a GI or liver disorder and will improve maternal care. ${ }^{9}$

The purpose of this study was to determine the most common indications for gastroenterology consultation in pregnancy at a high-volume obstetrics hospital.

\section{Materials and Methods}

A retrospective chart review was conducted of all consecutive outpatient gastroenterology consultations for pregnant women at the Center for Women's Digestive Disorders at Women and Infants Hospital, Providence, Rhode Island, over a 3-year period. The study was conducted with the approval of the Women and Infants institutional review board and in compliance with the Health Insurance Portability and Accountability Act (HIPAA). Women and Infants Hospital is one of the nation's leading specialty hospitals for women and newborns. The primary teaching affiliate of the Alpert Medical School of Brown University for obstetrics, gynecology, and newborn pediatrics, Women and Infants Hospital is the tenth largest obstetrical service in the country. More than 9700 deliveries take place in the hospital per year.

The Center for Women's Gastrointestinal Services is a section of the Department of Medicine at Women and Infants Hospital. Five thousand outpatient visits to the Center take place per year. In addition, the Center receives 400 inpatient consultations per year. A women's-only endoscopy unit is located within the Center wherein 2700 procedures take place per year.

A list of all completed outpatient visits for pregnant women between October 1, 2004, and October 17, 2007, at the Center for Women's Gastrointestinal Services was generated using the IDX Flowcast Application (GE Healthcare). These cases were identified using the scheduling search term "PCON" (Pregnancy Consultation), which is used to code new consultations for pregnant patients. Only outpatient consultations for new patients to the Center identified for the time period specified were eligible for the study. Established patients of the Center who became pregnant during the study period, women with multiple gestation pregnancies, and women whose pregnancies ended in miscarriage were excluded. For patients with more than one pregnancy during the study period, only the first pregnancy was reviewed.

Medical charts were reviewed to ascertain patient demographics, obstetrical history, referring source, indication for consultation, and final diagnoses (based on ICD-9 code). Testing generated as a result of GI consultation, change in management after consultation (defined as initiation or dis- continuation of a medication, change in dosage or route of an existing medication, recommendations for mode of delivery, referral to another clinical service, or recommendations for dietary changes), and need for GI follow-up during pregnancy or postpartum was also recorded. Patients with more than one indication for consultation had up to four indications recorded. Patients with multiple final diagnoses had up to four diagnoses included in the analysis.

\section{Results}

Four hundred six outpatient GI consultations in pregnancy were identified by the IDX Flowcast Application search. Cases were excluded as follows: (1) visit miscoding (patient not pregnant at the time of consultation or patient previously established at the Center at the time of pregnancy): 23, (2) multiple gestation pregnancy: 6, (3) pregnancy ending in miscarriage: $3,(4)$ second pregnancy during study period: 1 , and (5) patient chart missing or irretrievable: 14 . The remaining 370 cases were included in this analysis.

\section{Patient demographics}

Patients ranged in age from 14 to 51 years. The mean age ( \pm standard deviation [SD]) at referral was 28.7 years \pm 6.5 . The median gravida per patient was 2.0 (range 0-9), and median parity was 1.0 (range $0-8$ ). Primigravidas comprised $36.7 \%$ of patients. The mean gestational age at the time of referral was 21.3 weeks \pm 8.8 . Demographic data are summarized in Table 1.

\section{Referring source}

Obstetrician/gynecologists referred $70.1 \%$ of patients for a consultation. Other referring providers included internal

TABle 1. Characteristics of 370 OutPatient Gastrointestinal Consultations

\begin{tabular}{lr} 
Characteristic & Total No. (\%) \\
\hline Mean age \pm SD (years) & $28.7 \pm 6.5$ \\
Mean weeks gestation \pm SD (weeks) & $21.3 \pm 8.8$ \\
Median gravida (range) & $2(1-9)$ \\
Median parity (range) & $1(1-8)$ \\
Referring source & $261(70.5)$ \\
Obstetrician/gynecologist & $30(8.1)$ \\
Internist & $14(3.8)$ \\
Family practitioner & $13(3.5)$ \\
Other gastroenterologist & $14(3.8)$ \\
Midwife (obstetrical) & $6(1.6)$ \\
Midwife (family practice) & $5(1.4)$ \\
Emergency department physician & $1(0.3)$ \\
Maternalfetal medicine specialist & $21(5.7)$ \\
Other & \\
Main reason for referral & $239(64.6)$ \\
New GI symptoms in pregnancy & $94(25.4)$ \\
Worsening of preexisting GI & \\
$\quad$ symptoms in pregnancy & $53(15.1)$ \\
Recurrent GI symptoms & \\
$\quad$ in subsequent pregnancy & $11(3.0)$ \\
Advice on GI medication safety &
\end{tabular}

GI, gastrointestinal; SD, standard deviation. 
medicine providers $(8.1 \%)$, family practice providers $(5.4 \%)$, and other gastroenterologists (4.1\%) (Table 1).

\section{Indications for consultation}

Cases were categorized by main indication for consultation: $35.4 \%$ of consultations were for new GI symptoms arising in pregnancy, $24.4 \%$ for worsening of a preexisting GI disorder or preexisting GI symptoms, 15.1\% for GI symptoms recurring in a subsequent pregnancy, and 3.0\% for GI medication safety recommendations during pregnancy, lactation, or both (Table 1).

The presenting symptoms/initial diagnoses of the consultations are summarized in Table 2. The most common indications for consultation were viral hepatitis $(20.2 \%)$, nausea and vomiting $(18.9 \%)$, and abdominal pain (13.5\%).

\section{Final diagnosis}

The most common final diagnoses as a result of consultation were viral hepatitis $(17.8 \%)$, hyperemesis gravidarum $(15.1 \%)$, and gastroesophageal reflux (14.3\%) (Table 3). Constipation was the fourth most common diagnosis, comprising $13.0 \%$ of consultations. The majority of patients diagnosed with viral hepatitis had hepatitis B $(n=40)$, followed by hepatitis C $(n=23)$ and acute hepatitis A $(n=3)$. Diagnosis of viral hepatitis was based on serological testing or hepatitis $B$ DNA or hepatitis C RNA testing. Patients with hyperemesis

Table 2. Presenting Symptoms/Initial Diagnosis

\begin{tabular}{lc}
\hline Symptom/initial diagnosis $^{\text {a }}$ & No. of cases (\%) \\
\hline Viral hepatitis (chronic and acute) & $75(20.2)$ \\
Nausea and vomiting & $70(18.9)$ \\
Abdominal pain, not otherwise specified & $50(13.5)$ \\
Diarrhea & $36(9.7)$ \\
Constipation & $35(9.5)$ \\
Heartburn & $33(8.9)$ \\
Abnormal liver function tests & $33(8.9)$ \\
Hyperemesis gravidarum & $27(7.3)$ \\
Hematochezia & $19(5.1)$ \\
Inflammatory bowel disease (includes & $18(4.9)$ \\
ulcerative colitis, Crohn's disease, & \\
indeterminate colitis) & \\
Perianal disease (includes anal fissure, & $6(1.6)$ \\
$\quad$ hemorrhoids, rectal pain) & $6(1.6)$ \\
Insufficient weight gain & $5(1.4)$ \\
Hematemesis & $5(1.4)$ \\
Dysphagia & $4(1.1)$ \\
Pruritus & $4(1.1)$ \\
Irritable bowel syndrome & $3(0.8)$ \\
Nonviral hepatitis (acute and chronic) & $3(0.8)$ \\
Bloating/belching & $3(0.8)$ \\
Gallstone disease (includes biliary colic, & \\
choledocholithiasis, cholangitis & \\
and gallstone pancreatitis) & $2(0.5)$ \\
Peptic ulcer disease & $2(0.5)$ \\
Celiac disease & $2(0.5)$ \\
Nutritional deficiency, fatigue after & $2(0.5)$ \\
gastric bypass & $6(1.6)$ \\
Other & \\
\hline
\end{tabular}

${ }^{\mathrm{a}} \mathrm{Up}$ to four symptoms/initial diagnoses were recorded for each case. gravidarum were diagnosed based on the following findings: intractable nausea and vomiting beginning at $\leq 12$ weeks of gestation, ketonuria, and $>5 \%$ loss of prepregnancy body weight. A diagnosis of gastroesophageal reflux disease (GERD) was made clinically based on symptoms of heartburn (or pyrosis) or water brash. Lastly, constipation was diagnosed when patients reported having a bowel movement fewer than three times per week or stools were described as being hard, dry, small in size, and difficult to eliminate.

The pregnancy-unique liver diseases of intrahepatic cholestasis of pregnancy, acute fatty liver of pregnancy, and hemolysis elevated liver enzymes and low platelets (HELLP)

Table 3. Final Diagnosis

\begin{tabular}{|c|c|}
\hline Diagnosis ${ }^{\mathrm{a}}$ & No. of cases (\%) \\
\hline Viral hepatitis (acute and chronic) & $66(17.8)$ \\
\hline Hyperemesis gravidarum & $56(15.1)$ \\
\hline Gastroesophageal reflux & 53 (14.3) \\
\hline Constipation & 48 (13.0) \\
\hline Nausea and vomiting of pregnancy & $28(7.6)$ \\
\hline $\begin{array}{l}\text { Diarrhea (includes infectious } \\
\text { and noninfectious) }\end{array}$ & $27(7.3)$ \\
\hline Abdominal pain, not otherwise specified & $25(6.8)$ \\
\hline $\begin{array}{l}\text { Inflammatory bowel disease (includes } \\
\text { Crohn's disease, ulcerative colitis, } \\
\text { indeterminate colitis, and microscopic } \\
\text { colitis) }\end{array}$ & $23(6.2)$ \\
\hline $\begin{array}{l}\text { Abnormal liver function tests } \\
\text { of unknown etiology }\end{array}$ & $21(5.7)$ \\
\hline Helicobacter pylori gastritis & $18(4.9)$ \\
\hline Perianal disease & $13(3.5)$ \\
\hline $\begin{array}{l}\text { Gallstone and bile duct disease } \\
\text { (includes biliary colic, } \\
\text { choledocholithiasis, cholangitis) }\end{array}$ & $12(3.2)$ \\
\hline $\begin{array}{l}\text { Pregnancy-unique liver diseases (includes } \\
\text { intrahepatic cholestasis of pregnancy, } \\
\text { acute fatty liver of pregnancy, HELLP } \\
\text { syndrome) }\end{array}$ & $9(2.4)$ \\
\hline Nonviral hepatitis (acute and chronic) & $8(2.2)$ \\
\hline Heartburn & $7(1.9)$ \\
\hline $\begin{array}{l}\text { Hematemesis (includes Mallory Weiss } \\
\text { tear, esophageal varices) }\end{array}$ & $5(1.4)$ \\
\hline $\begin{array}{l}\text { Infectious colitis (includes Clostridium } \\
\text { difficile colitis) }\end{array}$ & $4(1.1)$ \\
\hline Pancreatitis & $4(1.1)$ \\
\hline Dyspepsia & $4(1.1)$ \\
\hline Cirrhosis & $4(1.1)$ \\
\hline $\begin{array}{l}\text { Esophagitis (includes reflux } \\
\text { and infectious) }\end{array}$ & $4(1.1)$ \\
\hline Dysphagia & $3(0.8)$ \\
\hline False positive viral hepatitis serology & $3(0.8)$ \\
\hline Infectious gastroenteritis & $2(0.5)$ \\
\hline Tietze's disease & $2(0.5)$ \\
\hline Benign colon polyps & $2(0.5)$ \\
\hline Peptic ulcer disease & $2(0.5)$ \\
\hline Hematochezia & $2(0.5)$ \\
\hline Preeclampsia & $1(0.3)$ \\
\hline Achalasia & $1(0.3)$ \\
\hline Radiation colitis & $1(0.3)$ \\
\hline Insufficient weight gain in pregnancy & $1(0.3)$ \\
\hline Other & 6 (1.6) \\
\hline
\end{tabular}

${ }^{a}$ Up to four diagnoses were recorded for each case.

HELLP syndrome, hemolysis elevated liver enzymes and low platelets syndrome. 
syndrome, which are often the most feared indications for GI consultation, accounted for only $2.4 \%$ of consultations. Consultation led to a change in diagnosis in $25.1 \%$ of patients.

\section{Management and follow-up}

As a result of consultation, $84.5 \%$ of patients underwent diagnostic testing. Laboratory testing was conducted in $83.5 \%$ of patients, diagnostic imaging in $25.4 \%$, and endoscopy in $6.5 \%$. Consultation led to a change in management in $78.6 \%$ of patients. GI follow-up during pregnancy was scheduled for $77.3 \%$ of patients and for $37.8 \%$ during pregnancy and postpartum.

\section{Discussion}

Most physicians agree that internists who specialize should know how to manage pregnant patients with medical problems specific to their specialty. ${ }^{10}$ For gastroenterologists, this is challenging, given that the many physiological, biochemical, and anatomic changes to the GI tract in pregnancy can produce a spectrum of disorders. Knowing which conditions are most likely to be encountered in practice is needed to focus educational efforts in pregnancy issues such that accurate diagnostic and therapeutic recommendations may be offered.

We found that GI conditions that complicate but are not unique to pregnancy comprised the most common indications for referral. Over $50 \%$ of consultations received were for viral hepatitis $(20.2 \%)$, nausea and vomiting (18.9\%), and nonspecific abdominal pain (13.5\%). Most patients with viral hepatitis were asymptomatic and diagnosed during routine prenatal care either during mandatory universal screening (hepatitis B) or after risk factor-based screening (hepatitis C). Most women referred for nausea and vomiting had symptoms extending beyond the first trimester or had signs and symptoms suggestive of more aggressive disease (e.g., hyperemesis gravidarum) or another GI disorder. Finally, most patients with abdominal pain were referred after pregnancyassociated gynecological disorders (such as ectopic pregnancy, miscarriage, or preterm labor) were ruled out.

It is notable that the most common indications for referral were for conditions or symptoms that are routinely encountered in gastroenterology practice. In the pregnant patient, however, their workup and management require special considerations. Differential diagnoses must include pregnancyunique conditions. In addition, ordering and interpretation of diagnostic tests must account for the normal biochemical and physiological changes of pregnancy in the case of routine laboratory studies and potential risks to the fetus in the case of endoscopy and diagnostic imaging studies. Lastly, the safety of medications during pregnancy and lactation must be known, given the need to initiate, discontinue, or change the dose of a medication in $6.2 \%$ of cases and to render advice on medication safety in $3 \%$ of cases.

In the majority of cases, GI consultation confirmed the initial diagnosis of the referring provider. However, despite a change in diagnosis in $25.1 \%$ of cases, $78.1 \%$ of patients did undergo a change in management as a result of consultation. Changes in management included medication initiation, discontinuation or dose/route change, delivery recommendations, referral to another clinical service, and recommendations for dietary changes. Therefore, similar to prior studies examining consultation practices in obstetrics, ${ }^{11}$ GI consulta- tion served an important role in patient management, moreso than in diagnosis. Our study was not designed to measure the impact of the management changes on maternal and neonatal outcomes; however, this is an important area for future study.

The Gastroenterology Leadership Council (GLC), comprised of the four main U.S.- based professional gastroenterology societies, has recognized the importance of pregnancy-related GI disorders and has made training in this area a required component of gastroenterology fellowship. The GLC has published an extensive list of specific pregnancy and childbearing issues about which trainees should be knowledgeable. ${ }^{12}$ Although comprehensive teaching on all these topics is ideal, multiple barriers, such as limited numbers of GI faculty with expertise in pregnancy issues and poor collaboration with obstetricians/gynecologists, limit the training process. ${ }^{13}$ As a result, gastroenterologists in training lack self-efficacy, or a sense of capability, in the evaluation and treatment of pregnant women, which may lead to delayed treatment or inappropriate care. Until the barriers to training are removed and comprehensive training is provided, educational interventions targeted toward the most common indications for consultation can fill the immediate need for expertise on pregnancy issues in gastroenterology.

Not unexpectedly, obstetrician/gynecologists comprised the largest referring source in our study, initiating $70.5 \%$ of consultations. The American College of Obstetrics and Gynecology has stated that consultation should be sought when the patient's needs go beyond the primary caregiver's education, training, experience, or available resources. ${ }^{14} \mathrm{Al}-$ though the frequency of GI consultation in pregnancy is not known, given the rising rates of delayed maternal childbearing and pregnancies complicated by chronic illness, it is probable that as obstetricians are stretched farther, they will request consultative services with greater frequency. At Women and Infants Hospital, we have experienced a stable number of newborn deliveries from 2004 to 2009 (mean \pm SD, $9286 \pm 258)$. In comparison, the frequency with which pregnant women have been seen in our GI clinic over this period has steadily increased. In 2006, there were 206 outpatient encounters for a new consultation in pregnancy or follow-up of a GI disorder in pregnancy at the Center for Women's Gastrointestinal Services. In 2009, this number was 699. Although the appropriateness of these visits was not assessed in this study, the greater than 3-fold rise in the number of visits over 3 years suggests there is an increasing need or demand for gastroenterologists' oversight of women during pregnancy. Based on trends at our center, we predict that providing consultative support to the primary healthcare providers of pregnant women will become an increasingly important function for gastroenterologists in the future.

Limitations of our study include that this is a single-center experience and may not reflect national trends in GI referral. Women and Infants Hospital is a tertiary care obstetrics hospital; therefore, patients seen at our institution may not mirror the general obstetrics population. In addition, Women and Infants is unique in that it is an obstetrics hospital with fulltime gastroenterologists on staff. The integration of gastroenterology into the clinical services at Women and Infants has created a stronger collaboration between gastroenterology and obstetrics than at most centers. This likely has influenced the volume and nature of consultations received. 


\section{Conclusions}

Although it is well established that pregnancy alters normal GI tract physiology, which in turn can precipitate the onset of new GI symptoms or exacerbate preexisting GI conditions, very few data on the actual indications for GI consultation in pregnancy exist. Our study highlights the most common indications for outpatient GI consultation at a high-volume, tertiary care obstetrics hospital. This lends focus to the issues that gastroenterologists must be prepared to manage during pregnancy. We found that GI disorders that complicate but are not unique to pregnancy are the most common indications for referral. It is incumbent upon gastroenterologists to know how to evaluate and manage these disorders in pregnancy so we may serve as a valuable resource to our colleagues and improve the quality of healthcare delivered to pregnant women.

\section{Acknowledgments}

We thank Steven Moss, M.D., and Colleen Kelly, M.D., at Brown University for their careful review of the manuscript. The project described was supported by Award Number K12HD055894 (S.S.) from the Eunice Kennedy Shriver National Institute of Child Health and Human Development. The content is solely the responsibility of the authors and does not necessarily represent the official views of the Eunice Kennedy Shriver National Institute of Child Health and Human Development or the National Institutes of Health.

\section{Disclosure Statement}

The authors have no conflicts of interest to report.

\section{References}

1. Martin JA, Hamilton BE, Sutton PE, et al. Births: Final data for 2006. Nat Vital Stat Rep 2009;57:1-102.

2. Carlson JA. The role of the medical consultant in pregnancy. Med Clin North Am 1989;73:541-555.

3. Fisher RS, Robert GS, Grabowski CJ, et al. Altered lower esophageal sphincter function during early pregnancy. Gastroenterology 1978;74:1233-1237.

4. VanThiel DH, Gavaler JS, Joshi SN, et al. Heartburn of pregnancy. Gastroenterology 1977;72:668-678.

5. Wald A, Van Thiel D, Hoechstetter L, et al. Effect of pregnancy on gastrointestinal transit. Dig Dis Sci 1982;27:1015-1018.
6. Ko CW, Beresford AA, Schulte SJ, Matsumoto AM, Lee SP. Incidence, natural history, and risk factors for biliary sludge and stones during pregnancy. Hepatology 2005;41: 359-365.

7. Bianchi DW, Zickwolf GK, Weil GJ, Sylvester S, DeMaria MA. Male fetal progenitor cells persist in maternal blood for as long as 27 years postpartum. Proc Natl Acad Sci USA 1996;93:705-708.

8. Von Rango U. Fetal tolerance in human pregnancy-A crucial balance between acceptance and limitation of trophoblast invasion. Immunol Lett 2008;115:21-32.

9. Coodley MK, Coodley GO, Levinson W, et al. Internal medicine training in ambulatory gynecology: A housestaff survey. Arch Intern Med 1993;153:2588-2592.

10. Spagnoletti CL, Rubio DM, McNeil MA. Internal medicine residents' preparedness to care for reproductive-age and pregnant women. Teach Learn Med 2007;19:257-263.

11. Bird JS, Taslimi MM, Gonzales AR, et al. Contribution of consultants to care of compromised pregnancies. J Natl Med Assoc 1996;88:97-100.

12. Gastroenterology Leadership Council: American Association for the Study of Liver Diseases, American College of Gastroenterology, AGA Institute, American Society for Gastrointestinal Endoscopy. Training the gastroenterologist for the future: The gastroenterology core curriculum. Gastroenterology 2003;124:1066-1104.

13. Saha S, Roberson E, Richie K, et al. Women's health training in gastroenterology fellowship: A national survey of fellows and program directors. Dig Dis Sci 2011 Jan 26 [Epub ahead of print]. PMID 21267780.

14. American College of Obstetrician and Gynecologists. Seeking and giving consultation. ACOG committee opinion No. 265. Obstet Gynecol 2007;109:1255-1259.

Address correspondence to: Sumona Saha, M.D. Department of Medicine

Division of Gastroenterology and Hepatology University of Wisconsin School of Medicine and Public Health UW Medical Foundation Centennial Building, Room 4224

1685 Highland Avenue Madison, WI 53705-2281

E-mail: ssaha@medicine.wisc.edu 
